\title{
21. Soziale Kohäsion in der Nachbarschaft
}

\author{
Sonja Nowossadeck \& Katharina Mahne
}

\section{Kernaussagen}

Ältere berichten im Jahr 2014 häufiger von engen Nachbarschaftskontakten als Jüngere: Die 70- bis 85-Jährigen berichten mit 51,6 Prozent am häufigsten enge Nachbarschaftskontakte, bei den 40- bis 54-Jährigen sind es nur 42,2 Prozent. Auch die Wohnlage ist bedeutsam für die Enge der Kontakte: Personen, die in als gehoben eingestuften Wohnlagen leben, berichten zu 49,1 Prozent enge Nachbarschaftskontakte - in mittleren Wohnlagen sind es 43,3 Prozent, in einfachen Wohnlagen nur 38,3 Prozent.

Der Anteil von Menschen mit engen Kontakten zu den Nachbarinnen und Nachbarn ist zwischen 1996 und 2014 stabil: Jeweils etwas weniger als die Hälfte aller 40- bis 85-Jährigen berichtet enge Nachbarschaftskontakte (1996: 44,5 Prozent, 2014: 45,6 Prozent). Frauen hatten 1996 noch etwas häufiger enge Nachbarschaftskontakte als Männer. Dieser Unterschied existiert im Jahr 2014 nicht mehr.

Ältere geben im Jahr 2014 im Durchschnitt weniger hilfsbereite Nachbarinnen und Nachbarn an als Jüngere: Im Jahr 2014 geben die 40- bis 54-Jährigen 4,9 hilfsbereite Personen in der Nachbarschaft an, die 55- bis 69-Jährigen 4,3 und die 70- bis 85-Jährigen 3,9. Zudem ist unter Personen mit Mobilitätseinschränkungen der Anteil derjenigen mit eingeschränkten Nachbarschaftsnetzwerken (d. h. keine oder nur eine hilfsbereite Person in der Nachbarschaft) mit 19,9 Prozent im Vergleich zu den nicht in ihrer Mobilität Eingeschränkten (10,4 Prozent) etwa doppelt so hoch.

Menschen, die in einfachen Wohnlagen leben, geben häufiger eingeschränkte Nachbarschaftsnetzwerke an: Insgesamt haben im Jahr 2014 11,1 Prozent der 40- bis 85-Jährigen eingeschränkte Nachbarschaftsnetzwerke (d. h. keine oder nur eine hilfsbereite Person in der Nachbarschaft). Bewohnerinnen und Bewohner einfacher Wohnlagen berichten zu einem Fünftel (21,4 Prozent) eingeschränkte Nachbarschaftsnetzwerke. Von den Personen, die in gehobenen Wohnlagen leben, sind nur 7,9 Prozent in dieser Situation.

Die Mehrheit der 40- bis 85-Jährigen weiß, was in der Nachbarschaft geschieht, nur wenige gestalten sie mit: Im Jahr 2014 gibt die Mehrheit (61,0 Prozent) der Menschen in der zweiten Lebenshälfte an zu wissen, was in der Nachbarschaft geschieht und etwas mehr als die Hälfte $(54,4$ Prozent) spricht mit Nachbarinnen und Nachbarn über das Geschehen in der Nachbarschaft. Nur relativ wenige (15,6 Prozent) bestimmen mit, was in der Nachbarschaft geschieht. Auch hier ist die Wohnlage wichtig: So geben 64,1 Prozent der 40- bis 85-Jährigen in gehobenen Wohnlagen und nur 53,7 Prozent der Personen in einfachen Wohnlagen an, über das Geschehen in der Nachbarschaft informiert zu sein. 


\subsection{Einleitung}

Es ist der Wunsch der meisten älteren Menschen, möglichst lange selbstständig zu Hause wohnen zu bleiben und vielen gelingt dies auch bis ins hohe Alter. Die selbstständige Lebensführung im vertrauten Wohnumfeld kann gerade mit zunehmendem Alter als Ausdruck von Autonomie verstanden werden (Oswald 1996). Es wächst jedoch auch das Risiko von sozialer Isolation, da Menschen in höheren Altersgruppen verstärkt an gesundheitlichen Einschränkungen leiden oder allein leben (Bromell \& Cagney 2013). Wenn beispielsweise Gehbeeinträchtigungen auftreten, können die Möglichkeiten zum Erhalt von selbstständiger Lebensführung und gesellschaftlicher Partizipation nicht nur von den unterstützenden Eigenschaften der Wohnung und des Wohnumfeldes abhängen, sondern auch von anderen nicht unmittelbar versorgungsrelevanten Aspekten wie etwa guten nachbarschaftlichen Beziehungen (Wahl 2001; Wahl \& Weisman 2003).

Nachbarschaften sind an räumliche Nähe und damit an das Wohnquartier und seine baulichen Gegebenheiten gebunden. Sie stellen ein Geflecht sozialer Beziehungen in diesem räumlichen Kontext dar. Für Ältere haben Nachbarschaften eine besondere Bedeutung, da sie hinsichtlich ihrer räumlich-sozialen Umwelten empfindlicher werden und eine eingeschränkte Adaptationsfähigkeit zeigen. Viele Ältere halten sich jeden Tag lange Zeit im Wohnquartier auf und sie haben eine große Zahl wohnbezogener Aktivitäten, daher hat eine funktionierende Nachbarschaft noch stärker als für jüngere eine wichtige Funktion für ihr Wohlbefinden (Beetz, Müller, J., \& F. Hüttl 2009; Oswald \& Konopik 2015). Andererseits kann diese Umwelt die Aktivitäten des Älteren stimulieren und sie befördern (Beetz et al. 2009: 126). Nach Befunden stadtsoziologischer Forschung haben heutige Nachbarschaften zwar im Vergleich zu vormodernen Gesellschaften Versorgungsfunktionen an den Sozialstaat abgegeben, aber auch wichtige Aufgaben behalten, wie Nothilfe, Kommunikation, Sozialisation und soziale Kontrolle (Rohr-Zänker \& Müller 1998).
Soziale Kohäsion in der Nachbarschaft bezeichnet den Zusammenhalt der Bewohnerinnen und Bewohner einer räumlichen Umgebung und umfasst das Vertrauen zu den Nachbarinnen und Nachbarn, gegenseitige Hilfe und Kooperation, eine gemeinsame Identität beziehungsweise ein Gefühl der Zugehörigkeit und daraus resultierendes Verhalten. In Nachbarschaften, in denen dieser Zusammenhalt funktioniert, kennen sich die Nachbarinnen und Nachbarn, sie vertrauen einander und teilen gemeinsame Werte (Müller 2008; van Dijk, Cramm, \& Nieboer 2013). Nachbarschaftsbeziehungen können soziale Unterstützung bereithalten oder den Zugang zu Versorgungsleistungen erleichtern. Eine Reihe von Studien konnte zeigen, dass eine hohe soziale Kohäsion in der Nachbarschaft als Puffer gegen negative Auswirkungen von Stress oder kritischen Lebensereignissen wirken kann (van Dijk et al. 2013), negative Auswirkungen des Alleinlebens oder des Lebens in Armut auf das subjektive Wohlbefinden puffert (Cramm, van Dijk, \& Nieboer 2012) beziehungsweise substanziell zu einer hohen Lebenszufriedenheit beiträgt (Oswald, Jopp, Rott, \& Wahl 2011). Damit ist die soziale Kohäsion in der Nachbarschaft besonders im Alter ein wichtiger Faktor für eine hohe Lebensqualität.

Während die Auswirkungen von sozialer Kohäsion schon relativ gut erforscht sind, ist für ihre Bestimmungsfaktoren noch wenig bekannt. Es gibt unterschiedliche Befunde zum Verhältnis von höherem Alter und Nachbarschaftsbeziehungen. Auf der Ebene des Individuums zeigt sich, dass Ältere (wie auch Verheiratete und höher Gebildete) eine höhere soziale Kohäsion berichten und dass auf der Ebene der Nachbarschaft vor allem das Sicherheitsempfinden von Bedeutung für den sozialen Zusammenhalt ist (van Dijk et al. 2013). Andererseits konnten Dijk, Cramm und Nieboer (2013) zeigen, dass höheres Alter auch ein Hemmnis für soziale Kohäsion sein kann. Als Ursache dafür führen die Autorinnen das mit dem Alter zunehmende Risiko kognitiver und physischer Einschränkungen an, die soziale Aktivitäten behindern. 
Zudem sind Ältere eher dem Risiko ausgesetzt, kleinere Netzwerke und weniger sozialen Austausch zu haben.

Höpflinger (2009) betont die Rolle von Nachbarschaftskontakten in Lebensphasen, in denen die unmittelbare Wohnumgebung besonders bedeutsam ist - zum Beispiel bei jungen Eltern mit Kleinkindern oder bei betagten Menschen wegen ihrer oft eingeschränkten Mobilität. Dabei können gute Nachbarschaftskontakte im Alter Sicherheit vermitteln und intergenerationelle Perspektiven eröffnen. Höpflinger betont aber auch, dass Nachbarschaftshilfe bei schweren Krisen oder Pflegebedürftigkeit primär kurzfristig von Nutzen ist, wogegen langfristige Pflege die Nachbarschaftshilfe sozial überfordert.

Ältere Menschen (und Familien) haben häufig mehr und intensivere Nachbarschaftskontakte als junge (und kinderlose) Menschen (RohrZänker \& Müller 1998). Die Erklärung dafür könnte sein, dass Ältere mehr Zeit als Jüngere in ihrer Wohnumgebung verbringen und daher Nachbarschaftskontakte intensiver pflegen können beziehungsweise stärker auf diese Kontakte angewiesen sind. Ältere Frauen und Männer haben unterschiedliche Voraussetzungen für die Kontaktpflege in der Nachbarschaft. Ältere Frauen sind häufiger alleinlebend als ältere Männer und sie verbringen weniger Zeit außer Haus (Nowossadeck \& Engstler 2013; Menning 2004). Für einen Teil dieser Frauen bedeutet das, dass sie am Nachbarschaftsleben nicht partizipieren, sich aus dem öffentlichen Leben zurückziehen und in der Folge auch für niedrigschwellige Hilfsangebote der Nachbarschaft weniger erreichbar sein könnten.

Van Dijk et al. (2013) beobachteten einen positiven Zusammenhang von Wohnstabilität und sozialer Kohäsion. Längere Wohndauern bewirken, dass sich Beziehungen in der Nachbarschaft entwickeln können, die Bindung an das Wohnumfeld stärker wird und damit der soziale Zusammenhalt in der Nachbarschaft wächst. $\mathrm{Zu}$ einem ähnlichen Ergebnis kommen Oswald et al. (2011), die ebenfalls einen Zusammenhang zwischen Wohndauer und Wohnortbindung konstatieren und die Qualität der Nachbarschaft sowie die wahrgenommene Bindung an die Nachbarschaft als Ressource für die Lebensqualität besonders im sehr hohen Alter betrachten. Dittmann und Goebel (2010) heben ebenfalls hervor, dass Menschen mit einem engen Nachbarschaftskontakt eine höhere Lebenszufriedenheit haben. Die Qualität der Wohnlage hat einen erheblichen Einfluss auf das Zusammenleben in der Nachbarschaft: So konnte gezeigt werden, dass gerade in prekären Nachbarschaften, in denen ein erhöhter Bedarf an sozialer Unterstützung besteht, der Zugang $\mathrm{zu}$ nachbarschaftlichen Unterstützungsnetzwerken erschwert ist. Bewohnerinnen und Bewohner benachteiligter Quartiere haben eher kleine Netzwerke ohne zureichende Ressourcen, zudem scheinen sich in diesen Quartieren unterschiedliche Bewohnermilieus voneinander abzugrenzen (Günther 2015).

Wie bringen sich die Personen unterschiedlicher Altersstufen in das Nachbarschaftsleben ein? Für höhere Altersgruppen werden eher subtile Formen sozialer Partizipation in der Nachbarschaft angenommen, während sich Jüngere häufiger aktiv einbringen. Befunde anderer Studien zeigten, dass sich Ältere häufiger über die Geschehnisse im Quartier austauschten, als dass sie diese aktiv beeinflussten. Will man die Partizipation im Quartier bis ins sehr hohe Alter unterstützen, sind also auch eher niedrigschwellige Formen sozialer Teilhabe $\mathrm{zu}$ fördern (Oswald, Kaspar, Frenzel-Erkert, \& Konopik 2013; Oswald \& Konopik 2015).

Im vorliegenden Kapitel werden die folgenden Fragen untersucht:

1. Wie eng sind die Kontakte zu den Nachbarinnen und Nachbarn und wie hat sich die Kontaktenge seit 1996 gewandelt?

2. Wie viele Nachbarinnen und Nachbarn haben Menschen in der zweiten Lebenshälfte, die sie um einen Gefallen bitten können?

3. Wie ausgeprägt ist die Partizipation am Nachbarschaftsleben? 


\subsection{Datengrundlage ${ }^{1}$}

Daten. Die analysierten Variablen zum Zusammenleben in der Nachbarschaft stammen aus den Angaben im schriftlichen Fragebogen des Deutschen Alterssurveys (DEAS) aus den Jahren 1996 und 2014, sofern die interessierenden Variablen in diesem Jahr erhoben wurden.

Enge des Kontakts. Die Enge des Kontakts wurde mit einer fünfstufigen Skala gemessen ( $1=$,sehr eng $/ 2=$,eng $/ 3=$,weniger eng'/ $4=$,nur flüchtig'/ 5 = ,gar kein Kontakt'). Diese Angaben wurden in eine dichotome Variable rekodiert: enger Kontakt = Antwortkategorien 1 und 2, kein enger Kontakt = Antwortkategorien 3, 4 und 5. Angaben zur Enge des Kontakts liegen für alle Erhebungen von 1996 bis 2014 vor.

Anzahl der hilfsbereiten Nachbarinnen und Nachbarn. Die Zahl der Nachbarinnen und Nachbarn, die man um einen Gefallen bitten kann, wurde erstmalig 2014 erhoben und liegt als metrische Größe vor. Daraus wurden zum einen Gruppenmittelwerte berechnet. Zum anderen wurden die Angaben in einer neuen Variable gruppiert, die folgende zwei Kategorien umfasst: $1=$,kein oder nur ein Nachbar, 2 = ,zwei und mehr Nachbarn'.

Partizipation innerhalb der Nachbarschaft. Dieser Aspekt wurde 2014 erstmals erhoben und über drei Items erfragt: Informiertheit über die Nachbarschaft („Ich bekomme mit, was in der Nachbarschaft geschieht."), Kommunikation in der Nachbarschaft („Ich rede mit den Nachbarn über das, was in der Nachbarschaft geschieht.") und Mitwirkung in der Nachbarschaft („Ich bestimme ein Stück weit mit, was in der Nachbarschaft geschieht."). Die Items haben eine vierstufige Antwortskala $(1=$,trifft genau zu'/ 2 = ,trifft eher zu'/ 3 = ,trifft eher nicht zu'/ $4=$,trifft gar nicht $\mathrm{zu}^{\prime}$ ). Für die Analyse wurden diese Angaben in eine dichotome Variable rekodiert: ,trifft $\mathrm{zu}^{\mathrm{c}}=$ Antwortkategorien 1 und 2, , trifft nicht $\mathrm{zu}^{\prime}=$ Antwortkategorien 3 und 4.

Für die Analyse der Partizipation in der Nachbarschaft wurde zudem ein Index berech-

1 Die Daten des DEAS können für wissenschaftliche Zwecke kostenlos beim Forschungsdatenzentrum des DZA (www.fdz-dza.de) bezogen werden. net. Dafür wurden die drei Dimensionen der Partizipation zusammen betrachtet und drei Gruppen unterschieden. Die ,Aktiven' haben in allen drei Dimensionen der Teilhabe angegeben, dass dies auf sie zutrifft. Den Gegenpol bilden die ,Zurückgezogenen', die für keine der Dimensionen angegeben haben, dass sie auf sie zutreffen.

Gruppierungsvariablen. Zur Prüfung von Altersunterschieden wurden - analog zur Schichtung der Stichprobe - drei Altersgruppen verwendet: 40 bis 54 Jahre, 55 bis 69 Jahre und 70 bis 85 Jahre. Weiterhin wurden Geschlechtsunterschiede und Gruppenunterschiede nach Wohnlage und Mobilitätseinschränkungen untersucht.

Wohnlage. Verwendet wurden die Interviewerangaben zur Einschätzung der Wohnlage („Wie schätzen Sie die Wohnlage insgesamt ein?" Antwortmöglichkeiten $1=$,eindeutig gehoben' bis $5=$,sehr einfach'). Die Variable wurde rekodiert in die Kategorien, gehobene Wohnlage ${ }^{\prime}=1$ und 2 , mittlere Wohnlage ${ }^{\star}=3$ und ,einfache Wohnlage $=4$ und 5 .

Mobilitätseinschränkungen. Mobilitätseinschränkungen wurden über ein Item aus dem SF-36-Fragebogen erfasst (vgl. Kapitel 8): „Sind Sie durch Ihren derzeitigen Gesundheitszustand bei diesen Tätigkeiten...?" Die Antwortkategorien sind $1=$, stark eingeschränkt', $2=$,etwas eingeschränkt', 3 = ,überhaupt nicht eingeschränkt'. Das in diesem Kapitel ausgewertete Item ist: "Fortbewegung außerhalb der Wohnung, z. B. Autofahren, Öffentliche Verkehrsmittel benutzen“. Diese Variable wurde rekodiert in die Kategorien, eingeschränkt ${ }^{c}=1$ und 2 , , nicht eingeschränkt ${ }^{\prime}=3$.

Analyse. In den Abbildungen und im Text sind gewichtete (gruppenspezifische) Mittelwerte oder prozentuale Verteilungen angegeben. Weiterhin wurden Geschlechtsunterschiede und Regionsunterschiede (Ost-/Westdeutschland) untersucht. Um Gruppenunterschiede auf Signifikanz zu testen, wurden in Abhängigkeit vom Skalenniveau binäre logistische Regressionen berechnet. Das genaue Vorgehen ist in Kapitel 2 beschrieben. 


\subsection{Enge des Kontakts zu den Nachbarn}

Nachbarschaftliche Kontakte gehören für Menschen in der zweiten Lebenshälfte zur Normalität. Im Jahr 2014 geben 45,6 Prozent der 40- bis 85-Jährigen enge Kontakte zu den Nachbarn an (Abbildung 21-1).

\section{Ältere berichten im Jahr 2014 häufiger von engen Nachbarschaftskontakten als Jüngere.}

Dabei berichten Ältere häufiger enge Kontakte als Jüngere: Die 70- bis 85-Jährigen haben mit 51,6 Prozent am häufigsten enge Nachbarschaftskontakte, bei den 40 - bis 54-Jährigen sind es nur 42,2 Prozent. Männer und Frauen unterscheiden sich in Bezug auf enge Nachbarschaftskontakte nicht (Abbildung 21-1). Einen Einfluss auf die Enge der Nachbarschaftskontakte hat auch die
Qualität der Wohnlage (Abbildung 21-1): Personen, die in als gehoben eingestuften Wohngegenden leben, berichten im Jahr 2014 zu 49,1 Prozent enge Nachbarschaftskontakte - in mittleren Wohnlagen sind es 43,3 Prozent, in einfachen Wohnlagen nur 38,3 Prozent. Es kann angenommen werden, dass gehobene Wohnlagen weniger Fluktuation und damit eine stabilere Bewohnerschaft haben. Dies sind gute Bedingungen, um enge Nachbarschaftsbeziehungen zu entwickeln.

Zudem zeigt sich ein Zusammenhang zwischen der Wohndauer und der Enge des Kontakts zu den Nachbarn: Menschen, die 30 Jahre und länger in ihrer Wohnung leben, berichten im Jahr $2014 \mathrm{zu}$ 56,0 Prozent enge Nachbarschaftskontakte, Personen mit weniger als zehn Jahren Wohndauer dagegen nur zu 35,0 Prozent (ohne Abbildung).

Abbildung 21-1: Anteile der Personen, die (sehr) enge Kontakte zu den Nachbarinnen und Nachbarn berichten, nach Alter, Geschlecht und Wohnlage, im Jahr 2014 (in Prozent)

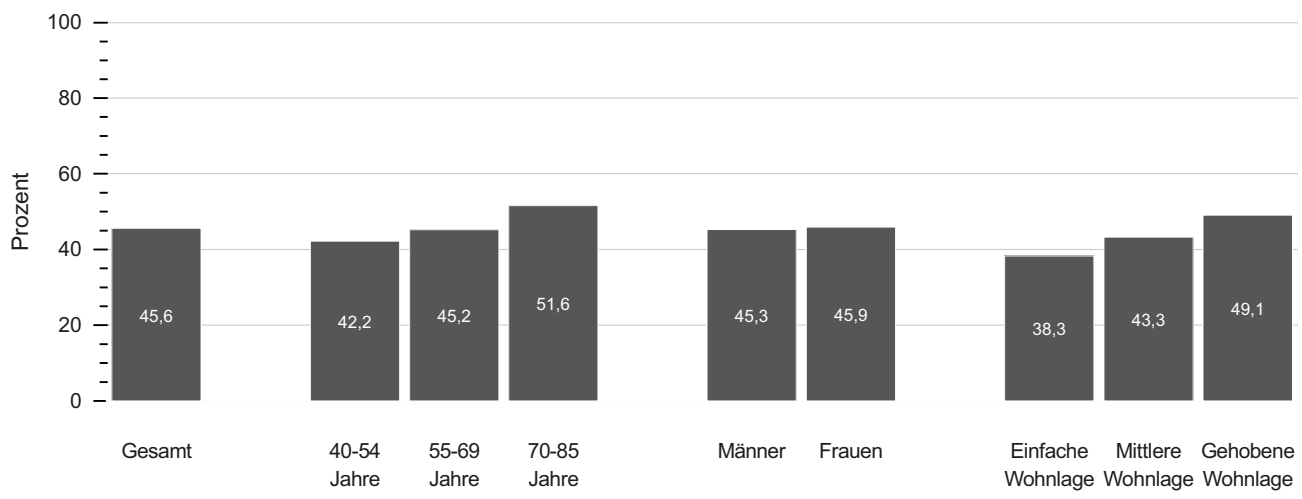

Quelle: DEAS 2014 ( $n=4.239$ für Alter und Geschlecht; $n=4.207$ für Wohnlage), gewichtete, gerundete Angaben; $(p<, 05)$.

Signifikanter Altersunterschied nur zwischen den 40- bis 54-Jährigen bzw. 55- bis 69-Jährigen und den 70- bis 85-Jährigen. Kein signifikanter Geschlechtsunterschied. Signifikante Unterschiede zwischen allen Wohnlagen.

Der Anteil von Menschen mit engen Kontakten zu den Nachbarinnen und Nachbarn ist zwischen 1996 und 2014 stabil.

Mit den Daten des DEAS kann man die Veränderung der Anteile von Menschen mit engen nachbarschaftlichen Beziehungen über einen Zeitraum von 18 Jahren verfolgen. Zwischen 1996 und 2014 ist die Kontaktenge in den Nachbarschaften stabil geblieben. Etwas weniger als die Hälfte aller 40- bis 85-Jährigen berichtet von engen Nachbarschaftskontakten: 2014 waren 
dies 45,6 Prozent, 1996 44,5 Prozent - die Veränderung dieser Anteile ist nicht signifikant (vgl. Tabelle A 21-1 im Anhang).

Bei keiner der drei Altersgruppen hat sich zwischen 1996 und 2014 der Anteil enger Nachbarschaftskontakte gewandelt. Unterschiede zwischen Älteren und Jüngeren - mehr enge Nachbarschaftskontakte in den höheren Altersgruppen - zeigen sich also zu jedem Zeitpunkt.
Bei den Männern ist der Anteil der Personen mit engen Nachbarschaftskontakten von 42,1 Prozent (1996) auf 45,3 Prozent (2014) angestiegen. Bei Frauen hat sich der Anteil nicht verändert (vgl. Tabelle A 21-1 im Anhang). Diese Entwicklung führt dazu, dass der 1996 noch vorhandene Unterschied zwischen Männern und Frauen im Jahr 2014 nicht mehr existiert.

\subsection{Anzahl hilfsbereiter Nachbarinnen und Nachbarn}

Enge Kontakte in der Nachbarschaft zu haben, ist für das Wohlbefinden im Wohnumfeld ein förderlicher Faktor. Aber können Personen in der zweiten Lebenshälfte im Alltag auch mit der Hilfe ihrer Nachbarinnen und Nachbarn in Form kleiner Gefälligkeiten rechnen? Mit der Anzahl der Nachbarinnen und Nachbarn, die man um eine Gefälligkeit bitten kann, wird die Hilfsbereitschaft zwischen Personen in der Nachbarschaft untersucht.

\section{Ältere geben im Jahr 2014 im Durchschnitt weniger hilfsbereite Nachbarinnen und Nachbarn an als Jüngere.}

Das Netzwerk von Nachbarinnen und Nachbarn, die man um einen Gefallen bitten kann, umfasst je nach betrachteter Gruppe im Jahr 2014 durchschnittlich 4,4 Personen. Die älteste Altersgruppe hat im Jahr 2014 im Durchschnitt eine hilfsbereite Person weniger in der Nachbarschaft als die jüngste: Die 40 - bis 54 -Jährigen berichten von 4,9 hilfsbereiten Nachbarinnen oder Nachbarn, die 55- bis 69-Jährigen von 4,3 und die 70 - bis 85 -Jährigen von 3,9. Personen, die in gehobenen Wohnlagen leben, nennen durchschnittlich 4,7 hilfsbereite Personen in ihrem Wohnumfeld, Personen in mittleren Wohnlagen 4,3 und die in einfachen Wohnlagen nur 3,4. Frauen und Männer unterscheiden sich hier wie auch bei der Enge der nachbarschaftlichen Kontakte - nicht (ohne Abbildung).

\section{Menschen, die in einfachen Wohnlagen leben, geben häufiger eingeschränkte Nachbarschaftsnetzwerke an.}

Interessant ist auch der Blick auf diejenigen Personen, die nur ein eingeschränktes Hilfspotenzial in der Nachbarschaft haben. Positiv ist: Es gibt generell nur sehr wenige Personen, die gar keine Nachbarinnen und Nachbarn haben, die sie um einen Gefallen bitten können. Im Jahr 2014 trifft dies gerade einmal auf 2,2 Prozent aller 40- bis 85-Jährigen zu. Alters- oder Geschlechtsunterschiede sind hier nicht zu beobachten. Aber Menschen, die in gehobenen Wohnlagen leben, berichten nur zu 1,4 Prozent gar keine hilfsbereiten Nachbarinnen und Nachbarn, während Menschen in mittleren (2,7 Prozent) und einfachen Wohnlagen (4,5 Prozent) deutlich häufiger ohne potenzielle nachbarschaftliche Unterstützung auskommen müssen (ohne Abbildung).

Die Beschreibung derjenigen, die nur eingeschränkt auf hilfsbereite Nachbarinnen und Nachbarn zurückgreifen können, umfasst im Folgenden auch die Personen, die nur eine einzige hilfsbereite Person in der Nachbarschaft haben, die sie um einen Gefallen bitten könnten. Ein Hilfsnetzwerk, das auf höchstens einer Person beruht, ist wenig stabil und nur eingeschränkt verlässlich, da diese Unterstützungsperson ausfallen kann. Insgesamt hat im Jahr 2014 etwa ein Zehntel (11,1 Prozent) der 40- bis 85-Jährigen keinen oder nur eine hilfsbereite Person in der Nachbarschaft (Abbildung 21-2). 
Die Jüngsten (40 bis 54 Jahre) sind mit 9,2 Prozent am seltensten von einem eingeschränkten Nachbarschaftsnetzwerk betroffen, bei den 70bis 85-Jährigen ist der Anteil mit 13,8 Prozent größer. Unterschiede zwischen Männern und Frauen bestehen dagegen nicht. In Ostdeutsch- land ist der Anteil derjenigen, die nur eine Person nennen oder gar keine nachbarschaftliche Unterstützung erfahren, mit 17,5 Prozent erheblich größer als in Westdeutschland (9,7 Prozent) (vgl. Tabelle A 21-2 im Anhang).

\section{Abbildung 21-2: Anteile der Personen mit 0 oder 1 bzw. 2 und mehr hilfsbereiten Nachbarinnen und Nachbarn, nach Alter, Geschlecht und Wohnlage, im Jahr 2014 (in Prozent)}

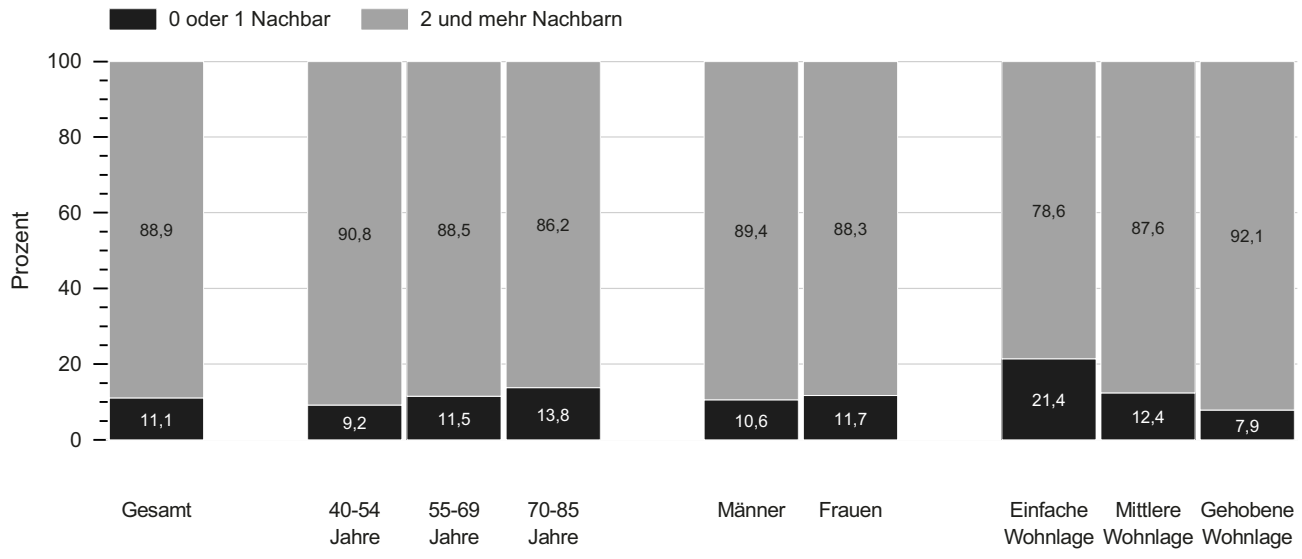

Quelle: DEAS 2014 (Alter und Geschlecht: $n=4.123$, Wohnlage: $n=4.091$ ), gewichtet, gerundete Angaben; $(p<, 05)$. Signifikante Unterschiede nur zwischen 40- bis 54-Jährigen und 70- bis 85-Jährigen. Kein signifikanter Geschlechtsunterschied. Alle Wohnlagenunterschiede signifikant.

Im Jahr 2014 ist die Anzahl hilfsbereiter Personen in der Nachbarschaft auch mit der Qualität der Wohnlage verknüpft. Dieser Zusammenhang könnte darin begründet sein, dass gehobene Wohnlagen in der Regel eine homogene und sozial gut situierte Bewohnerschaft haben. Das fördert ein Klima, in dem sich Vertrauen und ein offener Umgang miteinander entwickeln können. In deprivierten Wohnlagen mit einer oftmals heterogenen Bewohnerschaft und sozialen Problemlagen ist das nachbarschaftliche Miteinander schwieriger zu gestalten. Mehr als ein Fünftel der 40- bis 85-Jährigen in einfachen Wohnlagen (21,4 Prozent) hat kaum Möglichkeiten, auf nachbarschaftliche Unterstützung zurückzugreifen, auch wenn es nur um einen kleinen Gefallen geht (Abbildung 21-2). Das ist ein deutlich größerer Anteil als bei den Bewohnerinnen und Bewohnern gehobener Wohnlagen, von denen 7,9 Prozent nur eine oder gar keine hilfsbereite Person in der Nachbarschaft nennen.

Gerade bei Personen, bei denen aufgrund ihres Alters ein besonders großer Unterstützungsbedarf vermutet werden kann, erweist sich die Wohnlage als wichtiger Faktor: Von den 70- bis 85-Jährigen in einfachen Wohnlagen geben 26,9 Prozent an, keine oder nur eine Person im nachbarschaftlichen Unterstützungsnetzwerk $\mathrm{zu}$ haben. Gleichaltrige in gehobenen Wohnlagen sind nur zu 11,0 Prozent in dieser Situation (ohne Abbildung).

Gute nachbarschaftliche Kontakte sind besonders bedeutsam für diejenigen, die gesundheitliche Einschränkungen haben und daher auf Unterstützung im Alltag angewiesen sind. Personen, die Einschränkungen in ihrer Fortbewegung außerhalb der Wohnung berichten, haben häufiger das Risiko, nicht gut in Nachbarschaftsnetzwerke integriert zu sein (Abbildung 21-3). Unter den Menschen mit eingeschränk- 
ter Mobilität ist der Anteil derjenigen mit wenig potenzieller nachbarschaftlicher Hilfe mit 19,9 Prozent im Vergleich zu den nicht Eingeschränkten (10,4 Prozent) etwa doppelt so hoch. Dieser Unterschied ist in der jüngsten und in der ältesten untersuchten Gruppe zu beobachten: Bei den 40- bis 54-Jährigen haben 22,7 Prozent der in ihrer Mobilität Eingeschränkten nur eine oder keine hilfsbereite Person in der Nachbarschaft (im Vergleich zu 8,7 Prozent der Nichteingeschränkten), bei den 70 - bis 85 -Jährigen sind es 20,6 Prozent der in ihrer Mobilität Eingeschränkten im Vergleich zu 12,5 Prozent der nicht Eingeschränkten. Frauen, die sich außerhalb ihrer Wohnung nur eingeschränkt bewegen können, haben zu 21,4 Prozent ein reduziertes Nachbarschaftsnetzwerk, Männer mit den gleichen Problemen zu 17,7 Prozent. Es ist zu vermuten, dass Personen, die aufgrund ihrer gesundheitlichen Einschränkungen Probleme haben, außer Haus unterwegs zu sein, weniger gute Möglichkeiten haben, Kontakte im Wohnumfeld zu knüpfen und zu pflegen. Sie können daher zum Teil nur auf ein begrenztes nachbarschaftliches Hilfsnetzwerk zurückgreifen.

Abbildung 21-3: Anteil der Personen mit 0 oder 1 bzw. 2 und mehr hilfsbereiten Nachbarinnen und Nachbarn, nach Alter, Geschlecht und Mobilitätseinschränkungen außerhalb der Wohnung, im Jahr 2014 (in Prozent)

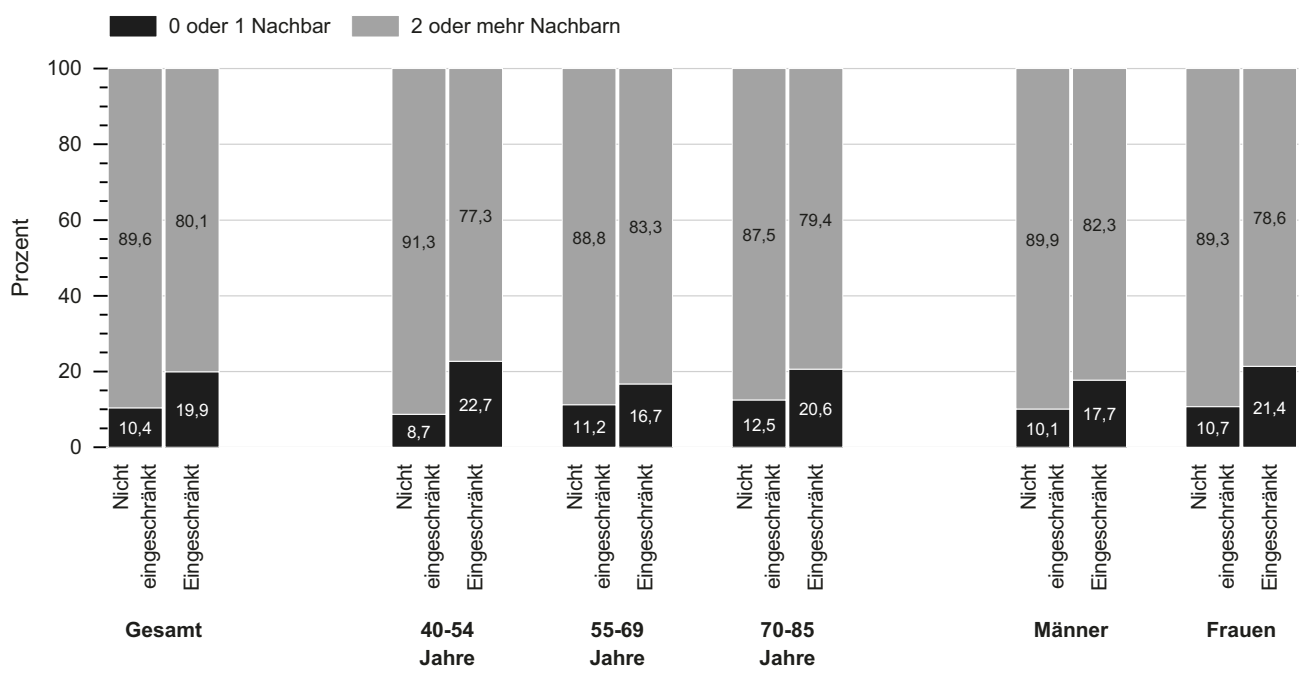

Quelle: DEAS 2014 ( $n=4.118)$, gewichtet, gerundete Angaben; $(p<, 05)$.

Alle Unterschiede zwischen nicht eingeschränkten und eingeschränkten Personen signifikant, außer in der Altersgruppe 55-69 Jahre. 


\subsection{Teilhabe und Mitwirkung in der Nachbarschaft}

Nachbarschaftskontakte bestehen nicht per se, sie müssen gepflegt und entwickelt werden. Nicht jeder will und kann sich in ein aktives Nachbarschaftsleben einbringen, nicht jede Nachbarschaft regt zur Teilhabe an. Für ältere Menschen allerdings hat eine funktionierende Nachbarschaft noch stärker als für jüngere eine wichtige Funktion für das Wohlbefinden, da Ältere viel Zeit in ihrem Wohnumfeld verbringen und viele Aktivitäten im engeren Umkreis stattfinden.

\section{Die Mehrheit der 40- bis 85-Jährigen weiß, was in der Nachbarschaft geschieht, nur wenige gestalten sie mit.}

Im Folgenden werden drei Aspekte analysiert, die in abgestufter Form Partizipation am nachbarschaftlichen Leben beschreiben: Informiertheit über Geschehnisse in der Nachbarschaft, Kommunikation über das, was in der Nachbarschaft passiert, sowie Mitgestaltung der Nachbarschaft. Je mehr Aktivität die einzelnen Formen der Partizipation erfordern, desto geringer sind die Anteile derjenigen, die diese Art der Teilhabe zeigen. Die Mehrheit (61,0 Prozent) der Menschen in der zweiten Lebenshälfte weiß im Jahr 2014, was in der Nachbarschaft geschieht, etwas mehr als die Hälfte (54,4 Prozent) spricht mit Nachbarinnen und Nachbarn über das Geschehen in der Nachbarschaft und nur relativ wenige (15,6 Prozent) bestimmen mit, was in der Nachbarschaft geschieht (Abbildung 21-4).
Wir sehen dabei durchgängig Unterschiede zwischen Männern und Frauen. Bei allen drei Aspekten der nachbarschaftlichen Partizipation sind Männer öfter beteiligt als Frauen. Sie geben häufiger an als Frauen, informiert zu sein über die Nachbarschaft, darüber mit Nachbarinnen und Nachbarn zu reden und mitzubestimmen, was in der Nachbarschaft geschieht. Dazu könnte der Umstand beitragen, dass Frauen vor allem im höheren Alter sich weniger oft außer Haus aufhalten als Männer, dass sie häufiger funktionale Einschränkungen haben, die ihre Mobilität behindern und dass sie sensibler auf ein (vermeintlich) unsicheres Wohnumfeld reagieren als Männer.

Beobachten können wir auch einen $\mathrm{Zu}$ sammenhang zwischen Wohnlage und nachbarschaftlicher Partizipation. Je gehobener die Wohnlage ist, desto mehr Menschen nehmen aktiv am Nachbarschaftsleben teil. So geben 64,1 Prozent der 40- bis 85-Jährigen in gehobenen Wohnlagen und 53,7 Prozent der Personen in einfachen Wohnlagen an, über das Geschehen in der Nachbarschaft informiert zu sein (Abbildung 21-4). 56,4 Prozent der Bewohner gehobener Wohnlagen und 48,4 Prozent der Bewohner einfacher Wohnlagen reden mit Nachbarinnen und Nachbarn darüber, was in der Nachbarschaft geschieht. Lediglich bei der Mitbestimmung dessen, was in der Nachbarschaft geschieht, zeigen sich keine Unterschiede nach Wohnlage. 
Abbildung 21-4: Anteile von Personen, für die Informiertheit, Kommunikation und Mitbestimmung in der Nachbarschaft (eher) zutreffen, gesamt, nach Alter, Geschlecht und Wohnlage, im Jahr 2014 (in Prozent)

a) Informiertheit über Nachbarschaft

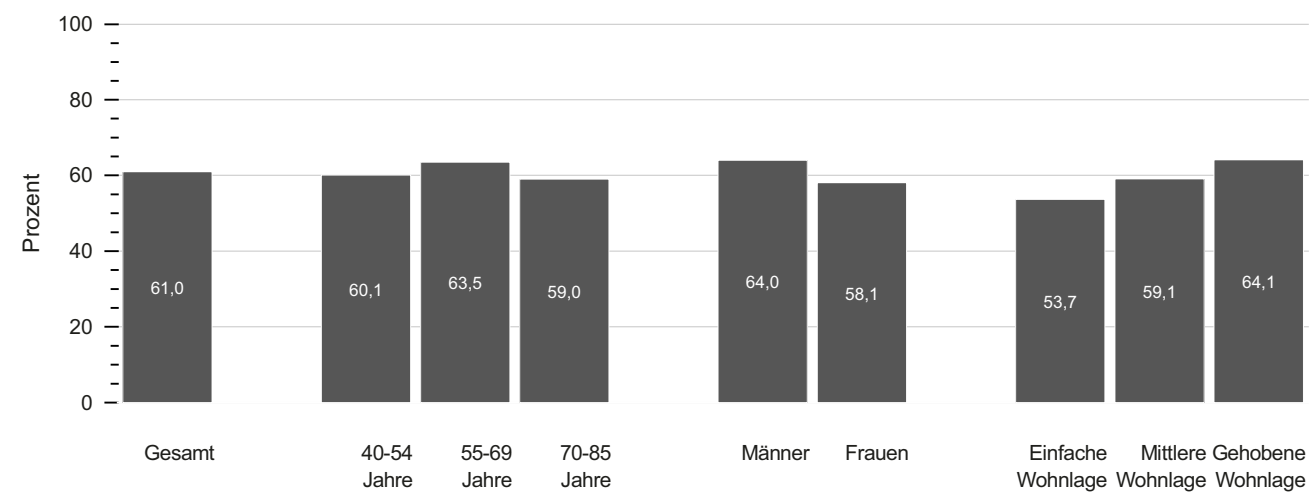

b) Kommunikation in Nachbarschaft

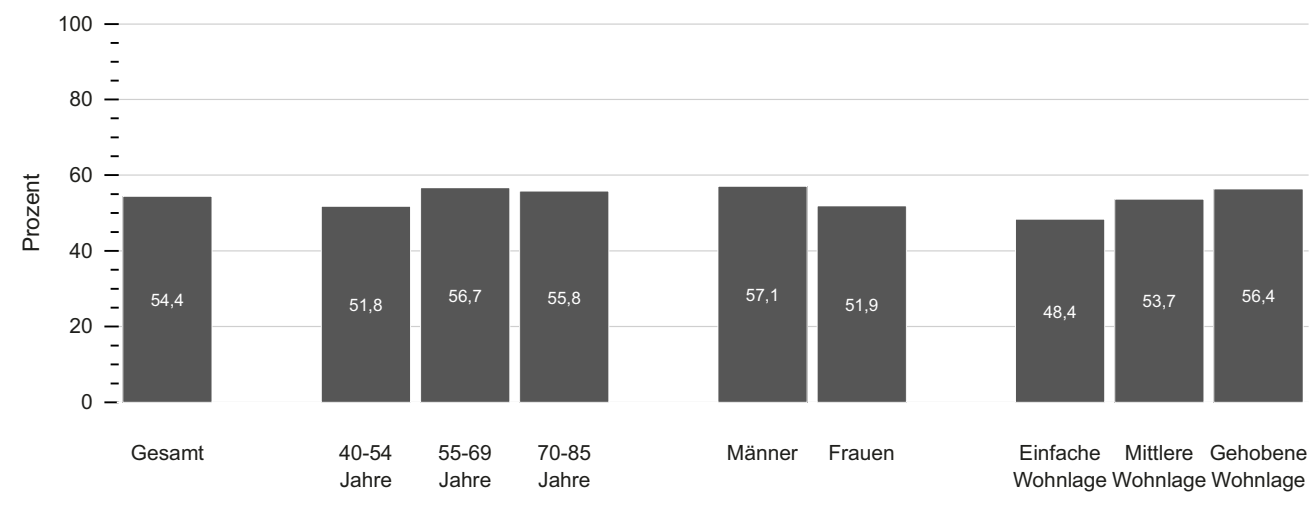




\section{c) Mitbestimmung in Nachbarschaft}

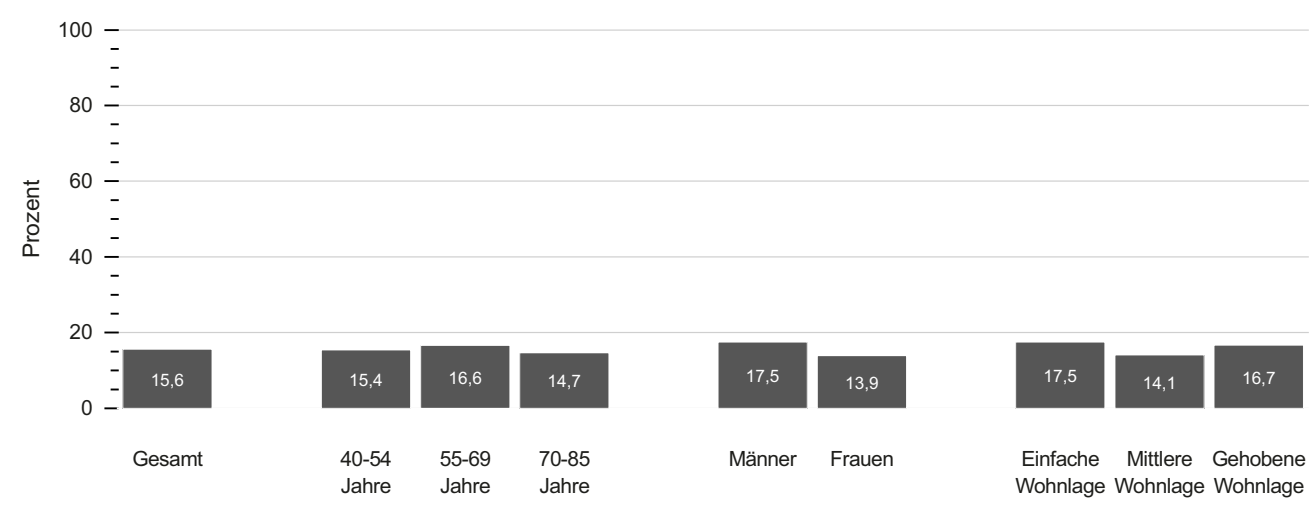

Quelle: DEAS 2014 ( $n=4.225$ für Informiertheit; $n=4.218$ für Kommunikation; $n=4.209$ für Mitbestimmung), gewichtet, gerundete Angaben; $(p<, 05)$.

a) Signifikante Altersunterschiede, außer zwischen 40- bis 54-Jährigen und 70- bis 85-Jährigen. Signifikante Geschlechts- und Wohnlageunterschiede. b) Signifikante Altersunterschiede, außer zwischen 55- bis 69-Jährigen und 70- bis 85-Jährigen. Signifikante Geschlechtsunterschiede. Signifikante Wohnlageunterschiede nur zwischen einfacher und gehobener Wohnlage. c) Keine signifikanten Altersunterschiede. Signifikanter Geschlechtsunterschied. Keine signifikanten Wohnlageunterschiede.

Betrachtet man die drei behandelten Aspekte der Teilhabe in der Nachbarschaft gemeinsam, zeigt sich, dass etwa ein Drittel (29,6 Prozent) vom Nachbarschaftsleben zurückgezogen lebt (Abbildung 21-5).

\section{Abbildung 21-5: Anteile der in Bezug auf die Nachbarschaft zurückgezogen Lebenden, gesamt, nach Alter, Geschlecht und Wohnlage, im Jahr 2014 (in Prozent)}

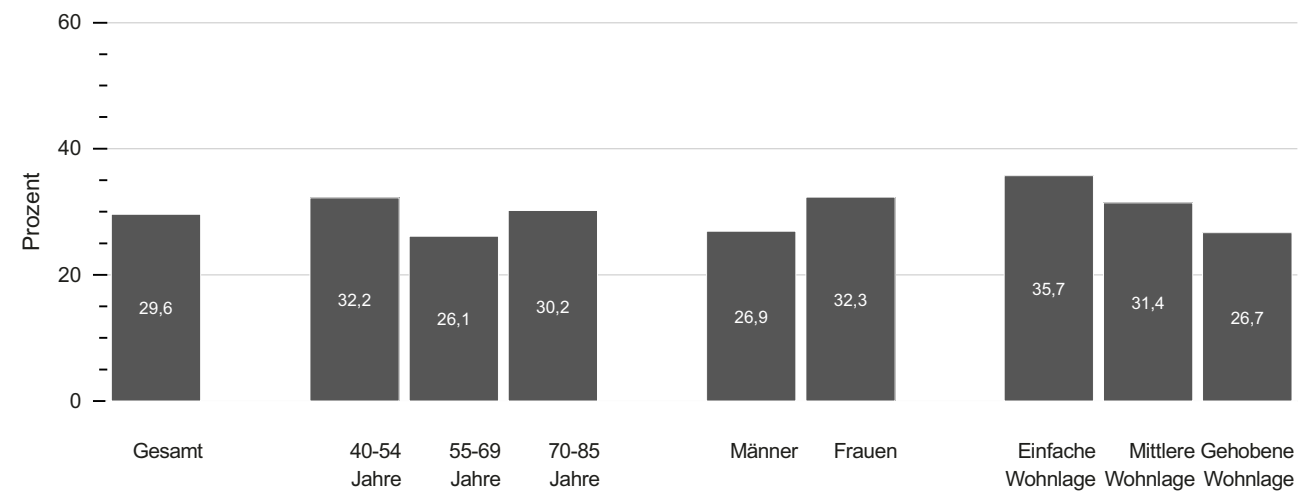

Quelle: DEAS $2014(\mathrm{n}=4.194)$, gewichtete, gerundete Angaben; $(p<, 05)$.

Signifikante Altersunterschiede außer zwischen den Altersgruppen 40-54 Jahre und 70-85 Jahre. Signifikante Geschlechtsunterschiede. Signifikante Wohnlageunterschiede außer zwischen mittlerer und einfacher Wohnlage.

Das bedeutet, die Person weiß nicht, was in der Nachbarschaft geschieht, kommuniziert nicht darüber und bestimmt das Nachbarschaftsle- ben auch nicht mit. Die Gründe dafür können vielfältig sein. Wer nicht an Nachbarschaften partizipiert, hat vielleicht andere Netzwerke, 
die das Leben bereichern, aber auch Zeit und Aufmerksamkeit fordern. Die ,Zurückgezogenen' finden sich häufiger unter den 40 - bis 54-Jährigen (32,2 Prozent) als unter den 55- bis 69-Jährigen (26,1 Prozent). Hierfür könnte die Erklärung zutreffen, dass die jüngere Altersgruppe in eine Reihe beruflicher, familiärer und anderer Netzwerke eingebunden ist und so das Leben in der Nachbarschaft kein so großes Gewicht hat. Frauen gehören mit einem knappen Drittel (32,3 Prozent) häufiger zu den ,Zurückgezogenen` als Männer mit etwa einem Viertel (26,9 Prozent). Dieser Unterschied ist vor allem bei den Älteren auffallend: Frauen im Alter von 55 bis 69 Jahren haben einen 6,8 Prozentpunkte höheren Anteil von ,Zurückgezogenen' als gleichaltrige Männer, bei den 70- bis 85-Jährigen beträgt diese Differenz sogar 10,4 Prozentpunkte, bei den 40 - bis 54-Jährigen dagegen nur 1,5 Prozentpunkte (vgl. Tabelle A 21-4 im Anhang). Die Erklärung dafür, dass sich vor allem Frauen der älteren Altersgruppen aus dem Nachbarschaftsleben zurückziehen, kann in ihrer Lebenssituation gefunden werden. Ältere Frauen sind seltener außer Haus unterwegs als ältere Männer und ein Teil dieser Frauen zieht sich aus dem öffentlichen Leben und damit auch aus der Nachbarschaft weitgehend zurück.

Wer in einer gehobenen Wohnlage wohnt, hat ein geringeres Risiko, zurückgezogen von der Nachbarschaft zu leben. Nur 26,7 Prozent der Bewohnerinnen und Bewohner gehobener Wohnlagen sind zur Gruppe der Zurückgezogenen zu zählen. Das sind weniger als in mittleren Wohnlagen (31,4 Prozent) und in einfachen Wohnlagen (35,7 Prozent).

Der Gegenpol zu den Zurückgezogenen sind die in Bezug auf die Nachbarschaft Aktiven, also diejenigen, die wissen, was in der Nachbarschaft geschieht, die darüber mit Nachbarinnen und Nachbarn reden und die auch das Nachbarschaftsleben mitbestimmen. Nur 13,2 Prozent der 40- bis 85-Jährigen gehören im Jahr $2014 \mathrm{zu}$ dieser Gruppe (vgl. Tabelle A 21-4 im Anhang). Diese Aktiven sind häufiger unter Männern (14,7 Prozent) als unter Frauen (11,9 Prozent) $\mathrm{zu}$ finden und sie leben häufiger in gehobenen Wohnlagen (14,5 Prozent) als in mittleren Wohnlagen (11,5 Prozent). Weitere Gruppenunterschiede, zum Beispiel in Bezug auf das Alter, sind bei den Aktiven nicht zu beobachten.

\subsection{Diskussion und Implikationen}

In der eigenen Wohnung zu leben, ist erklärtes Ziel der Mehrheit älterer Menschen. Damit das auch im hohen Alter und mit körperlichen Einschränkungen möglich bleibt, ist ein vielfältiges Unterstützungsnetzwerk erforderlich. Im sozialen Netzwerk spielen die Nachbarinnen und Nachbarn zwar hinter Familie, Verwandtschaft, Freundinnen, Freunden und Bekannten eine untergeordnete Rolle (Rohr-Zänker \& Müller 1998), sie haben aber eine wichtige ergänzende Funktion für soziale Kontakte und Unterstützung.

Die Ergebnisse des DEAS zeigen, dass diese nachbarschaftlichen Netzwerke existieren, funktionsfähig sind und dass die Enge nachbarschaftlicher Kontakte über die Jahre seit 1996 konstant hoch ist. Ein großer Teil der Älteren berichtet enge Kontakte zu den Nachbarinnen und Nachbarn und die große Mehrheit der Menschen in der zweiten Lebenshälfte kann sich an diese auch mit der Bitte um einen Gefallen wenden. Allerdings gibt es auch soziale Gruppen, die besonders gefährdet sind, nicht ausreichend in Nachbarschaftsnetzwerke integriert zu sein. Dazu gehören vor allem Menschen, bei denen ohnehin ein höherer Bedarf an Unterstützung durch das Umfeld vermutet werden kann, wie zum Beispiel hochaltrige und gesundheitlich in ihrer Mobilität eingeschränkte Personen. Diesen Personengruppen sollte über niedrigschwellige Nachbarschaftsangebote die Einbindung in das soziale Umfeld erleichtert werden.

Nachbarschaften funktionieren nicht (immer) von allein. In gehobenen Wohnlagen ist 
das nachbarschaftliche Unterstützungspotenzial ausgeprägter. Das heißt aber auch, dass in Gebieten, die als weniger privilegiert gelten, das Funktionieren dieser nachbarschaftlichen Netzwerke unterstützt werden sollte durch die Schaffung entsprechender Rahmenbedingungen. Infrastrukturelle Ausstattung und soziale Dienstleistungen im Wohnquartier sollten sich daran orientieren, wie Wohnquartiere ,nachbarschaftsfähig' gemacht werden können. Dazu gehört eine kommunikationsfreundliche Raumstruktur, eine kleinteilige wohngebietsnahe Versorgungsstruktur, die Vermittlung von Kontakten und nachbarschaftlichen Aktivitäten durch Gemeinwesenarbeit und die Übertragung von Zuständigkeiten und Selbstbestimmungsrechten (Rohr-Zänker \& Müller 1998).

So wichtig das Funktionieren von Nachbarschaften für die Integration und Teilhabe älterer Menschen auch ist: Die Erwartungen an die Leistungsfähigkeit von Nachbarschaften zur Unterstützung Älterer sollten nicht überfrachtet werden. Rohr-Zänker und Müller (1998) stellen fest, dass nachbarschaftliche Hilfeleistungen in der Regel nicht zeitintensiv oder verpflichtend sind und bezeichnen sie als ,Schönwetter-Beziehungen'. Auch im DEAS beziehen sich die Angaben zur Zahl hilfsbereiter Nachbarinnen und Nachbarn lediglich auf kleine Gefälligkeiten, nicht auf langfristige Unterstützungen, wie sie zum Beispiel in der Unterstützung und Pflege gebrechlicher Menschen erforderlich werden. Darüber hinaus verändern sich die Potenziale nachbarschaftlichen Zusammenlebens. Zunehmende Wohnmobilität und damit verbundene abnehmende Wohndauer, häufige Trennung von Arbeits- und Wohnort sowie steigende Erwerbstätigkeit von Frauen und Älteren können den Rahmen für nachbarschaftliches Engagement begrenzen.

Diese Problematik ist bekannt und es gibt eine Reihe von Initiativen mit dem Ziel, nachbarschaftliche Strukturen $\mathrm{zu}$ unterstützen und auszubauen. Dazu gehören zum Beispiel das Programm ,Soziale Stadt' oder auch neue Ansätze des Miteinanderlebens im ländlichen Raum, wie sie in verschiedenen Modellprojekten praktiziert werden. Diese Programme sollten in ihrer Wirksamkeit evaluiert und gestärkt werden.

\section{Literatur}

Beetz, S., Müller, B., J., B. K., \& F. Hüttl, R. F. (2009). Altern in Gemeinde und Region [Altern in Deutschland 5]. Stuttgart: Wissenschaftliche Verlagsgesellschaft.

Bromell, L., \& Cagney, K. A. (2013). Companionship in the Neighborhood Context: Older Adults' Living Arrangements and Perceptions of Social Cohesion. Research on Aging, 36(2), 228-243. doi: 10.1177/0164027512475096.

Cramm, J. M., Dijk, H. M. van, \& Nieboer, A. P. (2012). The Importance of Neighborhood Social Cohesion and Social Capital for the Well Being of Older Adults in the Community. The Gerontologist, 53(1), 142-52. doi: 10.1093/geront/gns052.

Dijk, H. M. van, Cramm, J. M., \& Nieboer, A. P. (2013). Social cohesion as perceived by community-dwelling older people: the role of individual and neighbourhood characteristics. International journal of ageing and later life, 8(2), 9-31.

Dittmann, J., \& Goebel, J. (2010). Your House, Your Car, Your Education: The Socioeconomic Situation of the
Neighborhood and its Impact on Life Satisfaction in Germany. Social Indicators Research, 96(3), 497-513. doi: 10.1007/s11205-009-9489-7.

Günther, J. (2015). Soziale Unterstützung und Nachbarschaft. Geschichte, Grundlagen, Perspektiven. In: C. Reutlinger, S. Stiehler \& E. Lingg (Hrsg.) Soziale Nachbarschaften (S. 189-200). Wiesbaden: Springer VS.

Höpflinger, F. (2009). Soziale Beziehungen im Alter-Entwicklungen und Problemfelder. Online: http://www. hoepflinger.com/fhtop/Soziale-Kontakte.pdf (zuletzt abgerufen 15.07.2016).

Menning, S. (2004). Die Zeitverwendung älterer Menschen und die Nutzung von Zeitpotenzialen für informelle Hilfeleistungen und bürgerschaftliches Engagement. In: Deutsches Zentrum für Altersfragen (Hrsg.) Expertisen zum Fünften Altenbericht der Bundesregierung (Bd. 5, S. 433-525). Berlin: LIT Verlag.

Müller, B. (2008). Soziale Kohäsion in der Nachbarschaft. Eine empirische Analyse von Einflussfaktoren auf Quartier-und Individualebene. Lizenziatsarbeit der Philoso- 
phischen Fakultät der Universität Zürich. Eingereicht am Soziologischen Institut der Universität Zürich bei PD Dr. Manuel Eisner.

Nowossadeck, S., \& Engstler, H. (2013). Familie und Partnerschaft im Alter [Report Altersdaten 03/2013]. Berlin: Deutsches Zentrum für Altersfragen.

Oswald, F. (1996). Hier bin ich zu Hause. Zur Bedeutung des Wohnens. Eine empirische Studie mit gesunden und gehbeeinträchtigten Älteren. Regensburg: Roderer.

Oswald, F., Jopp, D., Rott, C., \& Wahl, H. W. (2011). Is aging in place a resource for or risk to life satisfaction? Gerontologist, 51(2), 238-250. doi: 10.1093/geront/ gnq096.

Oswald, F., Kaspar, R., Frenzel-Erkert, U., \& Konopik, N. (2013). Hier will ich wohnen bleiben! Ergebnisse eines Frankfurter Forschungsprojekts zur Bedeutung des Wohnens in der Nachbarschaft für gesundes Altern. Frankfurt am Main: Goethe-Universität Frankfurt am Main und BHF-BANK-Stiftung.
Oswald, F., \& Konopik, N. (2015). Bedeutung von außerhäuslichen Aktivitäten, Nachbarschaft und Stadtteilidentifikation für das Wohlbefinden im Alter. Zeitschrift für Gerontologie und Geriatrie, 48(5), 401-407. doi: 10.1007/s00391-015-0912-1.

Rohr-Zänker, R., \& Müller, W. (1998). Die Rolle von Nachbarschaften für die zukünftige Entwicklung von Stadtquartieren. Expertise im Auftrag der Bundesforschungsanstalt für Landeskunde und Raumordnung. Oldenburg: Bundesamt für Bauwesen und Raumordnung. Online: http://stadtregion.net/fileadmin/downloads/Rolle_von_Nachbarschaften.pdf.

Wahl, H.-W. (2001). The living environment as a resource of old age. Integrated report on German expert contribution. Bonn: Bundesministerium für Familie, Senioren, Frauen und Jugend.

Wahl, H.-W., \& Weisman, G. D. (2003). Environmental gerontology at the beginning of the new millennium: Reflections on its historical, empirical, and theoretical development. The Gerontologist, 43(5), 616-627.

Open Access Dieses Kapitel wird unter der Creative Commons Namensnennung 2.5 International Lizenz (http://creativecommons.org/licenses/by/2.5/deed.de) veröffentlicht, welche die Nutzung, Vervielfältigung, Bearbeitung, Verbreitung und Wiedergabe in jeglichem Medium und Format erlaubt, sofern Sie den/die ursprünglichen Autor(en) und die Quelle ordnungsgemäß nennen, einen Link zur Creative Commons Lizenz beifügen und angeben, ob Änderungen vorgenommen wurden.

Die in diesem Kapitel enthaltenen Bilder und sonstiges Drittmaterial unterliegen ebenfalls der genannten Creative Commons Lizenz, sofern sich aus der Abbildungslegende nichts anderes ergibt. Sofern das betreffende Material nicht unter der genannten Creative Commons Lizenz steht und die betreffende Handlung nicht nach gesetzlichen Vorschriften erlaubt ist, ist für die oben aufgeführten Weiterverwendungen des Materials die Einwilligung des jeweiligen Rechteinhabers einzuholen. 